\title{
EXCALIBUR EN SICILE
}

\author{
Henri Bresc
}

La chronique de Roger de Hovedene raporte que, le 4 mars 1191 après plusieurs mois de conflits à Messine, Richard Coeur de Lion, venu à Catane depuis trois jours, avait reçu du roi Tancrède de nombreux dons, or, argent, chevaux, soieries, qu'il avait tous refusés, à l'exception d'un petit anneau. Tancrède devait lui donner aussi quatre grandes galères ussers, pour le transport de ses chevaux, et quinze galères, pour le Passage en Terre Sainte. En échange, en contre-don, le roi d'Angleterre donnait au roi de Sicile «cette épée admirable que les Bretons appellent Caliburne»'. Tout l'épisode, qui se conclut avec le retour de Richard à Messine, accompagné jusqu’à Taormine par son adversaire d'hier, baigne dans l'atmosphère arthurienne, chevaleresque et violente: Richard n'avait-il pas songé à la conquête de cette île de Sicile, si semblable, selon le mythe, ả la Bretagne, et, depuis novembre 1190, Arthur, son neveu, duc de la Bretagne armoricaine, n'était-il pas fiancé à la fille du roi de Sicile, dans le cadre d'une paix fragile que le don d'Excalibur venait maintenant sanctionner.

Cette épée, étroitement associée para le cycle romanesque au

${ }^{1}$ Roger de Hovedene, Chronica, éd. W. Stubbs, III, Londres 1870, p. 97. 
pouvoir même d'Arthur, et sans doute déjà présente dans les traditions orales mises en forme par Geoffroy de Monmouth, venait de très loin, d'une Bretagne légendaire et même d'un royaume de l'au-delà, d'où une archéologie pieuse l'avait tirée en 1190. Henri II avait ordonné des fouilles dans l'abbaye de Glastonbury, où les moines situaient l'île d'Avallon, séjour posthume d'Arthur. Il y avait un double intérêt; en retrouvant la tombe du roi légendaire, en donnant un corps enseveli au prince enlevé par les fées et conduit dans le paradis héroïque, Henri contribuait à éteindre la croyance populaire au retour d'Arthur, libérateur des Bretons et leur vengeur contre les Saxons. La monarchie normande, maintenant animée par les Plantagenêt, pouvait aussi se mouler dans le cadre de la légende, en hériter le prestige de la monarchie légendaire, hégémonique, européenne d'Arthur conquérant. La fouille, couronnée de succès, mettait au jour, symboliquement, un géant, conformément au topos qui veut que les fondateurs appartiennent à une humanité exceptionnelle par la taille et les vertus. Authentifiée par une inscription, la tombe, un tronc de chêne creux, contenait aussi les os de la useconde épouse», Guenièvre, et, sans doute aussi, l'épée qui rejoignait, en 1191, le trésor des rois de Sicile, bientôt uni à celui des Empereurs germaniques 2 .

On s'étonne cependant de voir Richard se séparer d'une arme légendaire, étroitement liée à la monarchie de Bretagne. II est vrai qu'un doute pouvait peser sur l'authenticité de la trouvaille: la légende, en vérité, et bientôt la tradition littéraire, associaient Excalibur à une Dame du Lac, l'épée, enchantée, était destinée à retourner à la fée des eaux ${ }^{3}$, et on sait que le thème celtique renvoie à une antique culture dont il est le reflet encore vif. $\mathrm{Ce}$ doute, cependant, si Richard le nourrissait, ne pouvait qu'être partagé par Tancrède: une même culture, épique et ro-

2 GIRAud le CAMBrien, De principis instructione, éd. G.F. Warnet, in Ope ra VIII, Londres 1891, pp. 126-128; l'inscription, sur une croix de plomb, portait «Hic jacet sepultus inclitus rex Arthurus cum Wenneuereia uxore sua secunda in insula Avallonian.

${ }^{3}$ Cf. JOËL H. GrisWARD, "Le motif de l'épée jetée au lac: la mort d'Arthur et la mort de Batradz”, Romania 90, 1969, pp. 289-340, 473-514. 
manesque, française, unissait les deux cours normandes. Mais il ne faut pas croire que la contradiction entre la légende et cette nouvelle réalité pouvait peser bien lourd dans le milieu des chevaliers et des princes; seuls les clercs curialistes pouvaient distinguer clairement et opposer les deux cultures. Le témoignage du merveilleux apporté à Glastonbury ranimait plutôt l'intérêt pour une tradition de toute façon multiple, polymorphe et chargée de significations changeantes. Cette arme était bien un cadeau royal, exceptionnel, inouï. Mais pourquoi en Sicile, à un roi lointain?

Arthur sur l'Etna...

L'histoire, en 1191, se moule sur le mythe, sur la tradition orale et sur la recherche des fondateurs, des origines glorieuses ou des prestiges ambigus. Il est clair qu'à la fin du xue siècle, la présence d'Arthur en Sicile est un fait massivement accepté. A. Graf a réuni, il y a près de cent ans, un gros dossier rassemblé dans le légendier des «folkloristes» du xume siècle" Goff a attiré récemment l'attention sur ces documents passionnants, où se fait jour la tentative d'une localisation sicilienne du Purgatoires: l'ensemble du dossier montre en effet l'hésitation des mythographes et la tentation d'associer cette présence d'Arthur et la topographie du «troisème lieu», rattachant, une fois de plus, la Sicile à l'ensemble britannique. C'est d'abord une légende recueillie par Gervais de Tilbury, un intellectuel normand d'Anglaterre, quand il séjournait dans l'île, un peu avant 1190, au service de la monarchie sicilienne, elle s'insère dans les relations intenses entre les deux monarchies, qui expliquent aussi la présence, dans l'oeuvre de Gautier Map son contemporain, de l'histoire de Nicola Pipi de Messine, «l'homme aquati-

4 ARTURO GrAF, "Artù sull'Etna», in Miti, leggende e superstizioni del Medio Evo II, Turin 1893, pp. 303-335.

5 Jacques Le Goff, La naissance du Purgatoire, Paris 1981, pp. 273-281 et 419-421; parallèlement à la localisation irlandaise. 
que». Dans les Otia imperialia, dédiés à Otton de Brunswick, Gervais greffe sur l'évocation, qui n'est pas localisée avec précision, de la «chasse sauvage» (la «mesnie Hellequin», d'ordinaire, mais ici rapportée à la Societas et familia Arturi), un conte, celui du cheval égaré et du serviteur porteur de message, qui lui a été confié personnellement par les «indigènes» et qui est confirmé - avec «admiration», c'est-à-dire stupeur-, par la trace matérielle des dons envoyés par Arthur ${ }^{6}$.

Le thème du conte permet en effet le passage aisé du monde des vivants à un autre monde, retranché derrière l'enfer brûlant des laves et des scories du volcan, mais, bien loin, à première vue, d'être effrayant, c'est une verte prairie, un paradis délicieux où s'élève un palais merveilleux. Cette plaine enchanteresse, c'est bien le séjour païen, celtique, d'Avallon, localisé en Sicile; le conte renvoie à Geoffroy de Monmouth et à la légende orale. le présence de Mordred et de Childéric ne peut venir déjà de la littérature française qui n'a pas encore élaboré de «Mort d'Arthur». C'est toujours un monde enchanté, marqué par le merveilleux: le palais, construit miro opere, purrait bien être l'oeuvre d'une puissance surnaturelle, comme la traduction de Jacques Le Goff nous invite à le considérer. Ce pourrait être, en particulier, l'oeuvre

6 Gervais de Trlbury, Otia imperialia, in Scriptores Rerum Brunswicensium, éd. Leibniz, Hanovre 1707, I, p. 291: «Hunc autem montem vulgares Mongibal appellant. In hujus deserto narrant indigenae Arturum magnum nostris temporibus apparuisse. Cum enim uno aliquo die custos palafredi episcopi Catanensis commissum sibi equum depulveraret, subito impetu lascivae pinguedinis equus exiliens ac in propriam se recipiens libertatem, fugit. Ab insequente ministro per montis ardua praecipitiaque quaesitus nec inventus, timore pedissequo succrescente, circa montis opaca perquiritur. Quid plura? arctissima semita sed plana est inventa; puer in spaciossimam planitiem jucundam omnibusque deliciis plenam venit, ibique in palatio miro opere constructo Arturum in strato regii apparatus recumbantem. Cumque ab advena et peregrino causam sui adventus percontaretur, agnita causa itineris, statim palafridum episcopi facit adduci, ipsumque praesuli reddendum, adjiciens se illic antiquitus in bello, cum Mordredo nepote suo et Childerico duce Saxonum, pridem commisso, vulneribus quotannis recrudescentibus, faucium dium mansisse quinimo, ut ab indigenis accepi, exenia sua ad antistitem illum destinavit, quae a multis visa et a pluribus fabulosa novitate admirata fuerunt». 
des fées, si l'on retient l'identification avec Avallon, le séjour immortel où les neuf soeurs, selon la Vita Merlini, ont emmené Arthur?. Mais on notera que le conte évite de citer le nom de l'aînée de ces soeurs, Morgane, comme si la chose était embarassante. Elle est pourtant connue de tous, elle fait partie de la tradition la plus antique, même si elle apparaît tardivement dans les textes littéraires. On remarquera encore qu'Arthur, bienveillant, hospitalier, généreux, gît ici sur un lit, car ses blessures se rouvrent chaque année, et c'est comme l'indice qu'il a été frappé aux jambes ou aux hanches.

L'Etna, siège d'un paradis païen qui renvoie aux légendes celtiques, sans l'ombre d'une malédiction apparente, est pourtant, aux yeux de tous, un enfer: depuis toujours, les volcans siciliens, sont, pour les Chrétiens, les bouches du royaume démoniaque. C'est ce que pense Pierre de Blois, retournant déconfit de l'aventure insulaire d'Etienne du Perche ${ }^{s}$. Reprenant un passage des Gesta Dagoberti ${ }^{9}$, Romuald de Salerne rappelle que l'ermite Jean a vu passer dans le ciel l'âme du roi franc portée et tourmentée par des démons «truculents»; la destination était Vulcano et le ciel ouvert pour permettre l'intervention des saints qui arrachent aux diables l'âme de Dagobert ${ }^{10}$. Une histoire

7 EDMON FARAL, La légende arthurienne. Etudes et documents II, Paris 1929 , p. 295.

${ }^{8}$ PIERre De BloIs, Epistolae, Patrologia Latina De Decano Palerniensi quem Rex Arcturus invitavit ad montem Gyber.

«Eo tempore quo Henricus Imperator subiugavit sibi Syciliam, in Ecclesia Palernensi quidam erat Decanus, natione ut puto Theutonicus. Hic cum die quadam suum qui optimus erat perdidisset palefredum, servum suum ad diversa loca misit ad investigandum illum. Cui homo senex occurrens, ait: Quo vadis, aut quid quaeris? Dicente illo, equum domini mei quaero; subiunxit homo: Ego novi ubi sit. Et ubi est? inquit. Respondit: In monte mons flammas evomit sicut Vulcanus. Stupente servo ad verba illius, subiunxit: Dic domino tuo ut ad dies quatuordecim illuc veniat ad curiam eius sollemnem. Quod si ei dicere omiseris, graviter punieris».

${ }^{9}$ L'iconographie du tombeau de Dagobert, élevé par saint Louis, à SaintDenis, illustrant le recit des Gesto, témoigne de Ja popularité du texte au Moyen-Äge.

10 Romuald de Salerne, Chronicon, éd. C.A. Garufi, Rome (RIS, VII, 1), 1914, p. 131. 
semblable, moins heureusement conclue, sera racontée bientôt à Césaire d'Heisterbach et datée de 1194-1196, pendant les trois années de la domination d'Henri VI sur la Sicile" ${ }^{11}$ une voix terrible annonce, sur l'Etna, Praepara focum magnum. Une autre voix demande, en écho, pour qui il faut allumer ce feu. C'est pour «notre cher ami le duc de Zähringen, qui nous a bien servi», Bertolph de Zähringen, cruel tyran et avide. Les auditeurs notent l'heure et le jour, ils vérifieront que c'est bien le jour et l'heure de la mort du duc.

Cette histoire s'insère dans un cycle, la douzième distinction, De Praemio Mortuorum, où plusieurs exempla, sur le même schéma, révèlent la présence diabolique à Vulcano ${ }^{12}$. C'est l'enfer, avec quelque chance de rachat, cependant, par les messes, les aumônes et les prières pour le bailli de Colmar. Os dicuntur esse inferni: le moine, qui répond aux questions du novice, laisse peu d'espérance; il évoque l'autorité des Dialogues de Grégoire le Grand, qui rapporte la malédiction de Théodoric, jeté dans les mêmes flammes: la localisation est banale, citée souvent et partagée avec le Vésuve. Ces anecdotes infernales encadrent le second conte qui met en jeu Arthur ${ }^{13}$ : parfaitement daté, des mêmes annés, rapporté par un témoin digne de foi, Godescalch, chanoine de Bonn, il a probablement une source française qui se trahit dans l'adjectif Palernensi (ce sont les Français qui nomment «Palerne» la capitale de la Sicile) et qui méconnait la géographie, ignorant la position de Catane au pied de l'Etna. C'est évidemment une réplique du conte de Gervais de Tilbury, mais assombrie et nocturne. Arthur n'y apparaît plus en personne, mais il a changé de fonction et de caractère; ni généreux, ni bienveillant, trop hospitalier cette fois, il est toujours roi de l'autre

" Cesatre d'Heisterbach, Dialogus miraculorum, éd. J. Strange, II, Cologne-Bonn-Bruxelles 1851, p. 325.

12 «De poena sculteri de Kolmere qui missus est in Vulcanum», p. 322; «De sculteto de Leggenich qui in eundem montem missus est», p. 323; «De Brunone de Flitert qui in eundem Vulcanum proiectus est», p. 323.

${ }^{13}$ Ibid. pp, 324-325: Reservus servus, quae audivit domino suo exposuit cum timore tamen. Decanus ad curiam Arcturi se invitatum audiens et irridens, infirmatus die praefixa mortuus est. 
monde, mais il est maintenant le roi des morts, qui convoque ses futurs sujets à sa cour et leur fixe un terme indépassable. Son royaume est un enfer, sans doute, mais Césaire n'a pas senti qu'il différait du monde diabolique qui peuple la douzième distinction. C'est un enfer sans flammes, ni démons. A côté des bouches qui conduisent à la demeure des dammés, la culture recueillie par nos folkloristes médiévaux avait donc placé tour à tour un paradis ambigu et verdoyant, et un autre monde glacé.

Déjà Gervais avait témoigné de la contamination entre le roi Arthur et Herla, l'autre roi breton, nocturne, maudit, patron de la «chasse sauvage»" Etienne de Bourbon évoque à son tour les chasseurs nocturnes, de familia Allequini vel Arturi; et il rapporte une troisième version du conte ${ }^{15}$. S'il évoque le Purgatoire nommément, c'est pourtant un royaume plus infernal encore

14 JEAN-CLAUdE SCHMITT a étudié particulièrement, dans «Temps, Folkiore et Politique au XIIe siècle", Le Temps chrétien de la fin de l'Antiquité au Moyen-Äge, Ille-XIIfe siecle, Paris 1984, pp. 489-515, le traitement du thème d'Herla par Gauthier Map.

${ }^{15}$ A. LECOY DE LA MARCHE, Anecdotes historiques, légendes et apologues tirés du recueil inédit d'Etienne de Bourbon, dominicain du xule siècle, Paris 1877 , p. 32: «Item audivi a quodam fratre Apulo, Johanne dicto, qui hoc dicebat in partibus suis accidisse, quod, cum quidam monte juxta Vulcanum, ubi dicitur locus purgatorii, prope civitatem Cathenam, quereret equum domini sui, inveniret, ut sibi visum est, civitatem quamdam, cujus erat hostiolum ferreum, et quesivit a portitore de equo quem querebat: qui respondit quod iret usque ad aulam domini sui, qui vel redderet eum vel doceret; et adjuratus ab eo portitor per Deum quod diceret ei quid ageret, dixit ei portitor quod caveret ne comederet de aliquo ferculo quod ei daretur. Videbatur ei quod videbat per vicos illius civitatis tot homines quot sunt in mundo, de omni gente et artificio. Transiens per multas aulas, venit in quamdam, ubi videt principem suis circumvallatum; offerunt ei multa fercula: non vult de eis gustare; ostenduntur ei quatuor lecti, et dicitur ei quod unus eorum erat domino suo paratus, et alii tres trium feneratorum. Et dicit ei princeps ille quod assignabat diem domino suo talem peremptoriam et tribus dictis feneratoribus, alioquin venirent inviti; et dedit ei ciphum aurcum, coopertum copperculo aureo. Dicit ei ne illum discooperiret, sed illum in hujus rei intersignum presentaret domino suo, ut biberet de potu suo. Equus suus ei redditur; reddit, implet jussa: cifus aperitur, flamma ebullit, in mari cum cifo proicitur, mare inflammatur. Hi quatuor, licet confessi fuissent (ex timore solo, et non vere penitentes) die sibi assignata, rapiuntur super quatour equos nigros». 
que découvre le serviteur, à la recherche du cheval de son maître. Arthur n'est plus nommé, son hospitalité est maintenant un piège brûlant. Laccepter, manger à sa table, c'est brûler pour l'éternité. Dans ce monde des morts, aussi peuplé que le monde des vivants, la place des maudits est réservée et le signe qu'on confie au serviteur est un signe de feu, qui enflamme la mer. Quatre chevaux noirs, "psychopompes", comme le fait noter J. Le Goff, viendront, le jour dit, emmener les usuriers et le maître à leur convocation. Cette monarchie des morts va bientôt accueillir le plus illustre des monarques, Frédéric II, persécuteur des Franciscains, et qui y conduit une armée de chevaliers maudits «de bronze incandescent ${ }^{16}$. Image négative, cléricale, mais non sans grandeur, qui relie, encore une fois, le mythe et l'histoire.

Ce caractère sombre, nocturne, infernal d'Arthur s'est donc accentué de conte en conte: l'Etna s'est «infernalisé», comme l'avait noté. A. Graf, sans doute sous la pression de la culture religieuse des clercs, qui l'a emporté sur l'image fraîche et claire du paradis celtique. Il est vrai que l'on avait noté dans ce dernier plus d'une ambiguité, et que le personnage d'Arthur luimême, depuis toujours, hésitait entre deux définitons et deux figures. A Modène, sur la Ghirlandina, aux côtés de Roland et de David, J. Stiennon et R. Lejeune identifient un Arthur positif, champion du bien ${ }^{17}$ : il terrasse un animal fabuleux. Echo à ces sculptures, datables entre 1169 et 1179 , la mosaïque de la $\mathrm{Ca}$ thédrale d'Otrante, où Arthur, Rex Arturus, est monté sur un animal monstrueux et cornu, un bouc à bec d'aigle. Mais là, l'équivoque n'est pas possible: le roi n'a pas terrassé l'animal, il le

16 ThOMAS DE ECCLESTON, Liber de adventu Fratrum Minorum in Angfiam, MGH SS 28, p. 568: hístoire rapportée par F. Mansuetus, venu en Angleterre en 1258: «Dixit etiam quod quidam frater stans in orto in oratione in Cicilia vidit maximum exercitum quinque milia militum equitum intrantem mare; et crepuit mare, quasi essent omnes ex ere candente; et dictum est ei ab uno eorum quod fuit Fredericus imperator, qui ivit in montem Ethne: nam eodem tempore mortuus est Fredericus".

17 JACQUES STIENNON et RITA LEJEUNE, «La légende arthurienne dans la sculpture de la cathédrale de Modène», Cahiers de civilisation médiévale VI, 1963, pp. 281-296. 
chevauche, armé d'une massue ${ }^{18}$. L'incarnation du Bien, telle qu'elle est apparue à Modène, à l'archivolte du portail de la Pescheria, où le roi délivre sa première épouse, Winlogee, des forces du mal, n'est done pas universelle. L'Etna a accueilli un Arthur ambivalent et la coexistence de deux cultures va maintenir deux images siciliennes du roi breton.

\section{...et la Fée Morgane}

L'image positive de la vallée paradisiaque où l'on ignore la mort n'est pas effacée pour toujours par la multiplication de ces anecdotes cléricales, qui ont infernalisé l'Etna. Cette vallée enchantée, elle est d'abord présente dans la littérature française de Sicile: dans la Bataille Loquifer ${ }^{19}$, écrite par Graindor de Brie, vers 1170, Rainouart, à la recherche de son fils Maillefer, est enlevé par les trois fées qui le portent en Avallon, où il rencontre Roland, Gauvain, Ivain, Arthur, «la gent faé et de cest sicle venu et trespassé». De l'aînée des trois, Morgane, amante passionnée, jalouse, vindicative et nettement polyandre, Rainouart engendrera le diable Corbon: luxure et magie, richesse et beauté débouchent dans la laideur ${ }^{20}$. Avallon, dans cette chanson, n'est pas loin de la Sicile et une localisation méridionale ne choque pas: vers la fin du xive siècle, l'auteur de Bâtard de Bouillon n'hésitera pas à placer au delà de la Mer Rouge, le pays de «Faerie», «la terre Artus et Morgue la jolie», un autre paradis sensuel «avec mille fées que les corps ont jolis», où le temps passe sans qu'on s'en aperçoive. Très tôt, c'est même en Sicile et précisément sur l'Etna que la littérature situe le séjour de Morgane.

Dans le Parzifal de Wolfram von Eschenbach, vers 1210, l'itinéraire de Tevrizent associe à la montagne d'Agremontin, le mont

${ }^{18} \mathrm{Cf}$. l'analyse de KURT WAIS, «Morgain amante d'Accalon et rivale de Guenièvre', Bulletin bibliographique de la Société internationale Arthurienne 18, 1966, pp. 137-149.

19 Éd. MONICA BARNETT, Oxford 1975.

${ }^{20} \mathrm{Cf}$. JeanNe Wathelet-Willem, «La fée Morgain dans la Chanson de Gesten, Cahiers de civilisation médiévale XIII, 1970, pp. 209-239. 
de Morgane, breg ze Famorgân"2 et dès 1180, dans Jaufré, la fée, à peine dissimulée, se présente: «Jeu sui la fada del Gibel», c'est-à-dire de l'Etna, que tout le Moyen Age appelle Mongibe1lo. Elle sera «la fée de Mongibel» dans le Chevalier du Papegau. Sa seigneurie sur l'Etna est clairement affirmée vers 1250 dans le roman Floriant et Florete ${ }^{22}$, qui illustre les thèmes de la dame d'un paradis terrestre doté de l'immortalité. Sensuelle encore, mais ici mère aussi, adoptive et tendre, Morgane veille, dans son domaine du Mongibel, sur Floriant, fils du roi de Sicile Elyadus, assassiné par le sénéchal félon. Bonne mère, elle fait de lui un chevalier parfit, «enfances féeriques» qui rappellent celles de Lancelot, celles de Maugis d'Aigremont, en Sicile, auprès d'Oriande. Elle doit encore le laisser partir, mais c'est sur son navire enchanté, sans rameur; elle est en effet aussi naïade nymphe marine $^{23}$. Les exploits de Floriant le ramènent dans l'île, empereur de Constantinople, roi de Sicile et mari de Florete, mais, un jour, à la poursuite d'un cerf blanc, Floriant pénètre dans le château de Morgane. Le cerf est redevenu la belle fée, assise sur un lit, qui lui révèle que ce jour était le terme de la vie terrestre de Floriant; il demande et obtient que Florete partage l'immortalité terrestre à laquelle participera bientôt Arthur.

Cette seigneurie sur l'Etna n'était sans doute pas ignorée quand Gervais interrogeait les gens de Catane; elle sera reprise dans la Faula de Guillem de Torroella, en $1375^{24}$, et elle débouche sur une géographie arthurienne inattendue de la Sicile: vers 1230, Frédéric II fait construire un château près de Castelvetrano, sur le Modione. On lui donne le nom de Bellurepar, c'est-à-dire Bel Repaire, le château où Morgane, dans les Enfances Gauvain ${ }^{25}$,

21 ROGER SH. LOOMIS, «Morgain la fée in oral tradition», Romania 80. 1959, pp. $337-367$.

22 Ed. Harry F. Williams, Ann Arbor-Londres 1947.

${ }^{23}$ Cf. RugGero M. RugGieri, «Morgain la fée: un personnage et un miragem, Bulletin bibliographique de la Société Internationale Arthurienne 18, 1966, pp. $170-171$.

24 Éd. GaBriel Llabres, Cançoner dels comtes d'Urgell, Barcelone 1906, pp. 29-148, sans localisation précise cependant.

25 P. MEYER, «Les Enfances Gauvain», Romania 39, 1910, pp. 1-32; Mor- 
accouchait de Gauvain, fils de ses amours avec Lot d'Orcaine. Plus tard encore, vers 1370 , un grand seigneur sicilien, Vinchiguerra Aragona, nomme Goiosa Guardia, la Joyeuse Garde, une villeneuve implantée dans le terroir de l'évêque de Patti ${ }^{26}$, claire référence à la Douloureuse Garde, rebaptisée par Lancelot «Joyeuse» et où il mène en effet, avec Guenièvre, joyeuse vie, en attendant de soutenir le siège dirigé par Arthur. Cette association entre légende arthurienne et Sicile, banale et sans doute très ancienne, $a$, on le notera, un caractère aristocratique très marqué. La noblesse sicilienne n'est pas rebutée par les aspects sensuels et païens des épisodes invoqués.

Le thème de la vallée enchatée et du paradis sensuel des fées n'a pas été localisé exclusivament dans l'île: Antoine de la Salle ${ }^{27}$ enregistre, vers 1420 , un ensemble de légendes qui localisent le Paradis de la Reine Sibylle dans la montagne de Norcia et les histoires des chevaliers qui s'y sont perdus - le chevalier allemand à qui Innocent VI ou Urbain V a refusé le pardon, l'oncle de Gaucher de Ruppes, le seigneur gascon de Pacss'échelonnent entre 1350 et 1380, tandis qu'Andrea de Barberino insère le Mont de la Sibylle dans le Guerino Meschino, en $1391^{28}$. Ce paradis infernal, rêve de volupté et mystère de perdition, oủ le temps s'écoule sans viellissement ni impressión de durée, et où tous les plaisirs sont prodigués par des fées, hérite le caractère d'Avallon- et de l'Avallon etnéen de Gervais. Il est encore plus inquiétant: au coeur d'une montagne, souterrain, c'est l'antichambre de la damnation; le vendredi soir, et jusqu'au soir

gane y est appelée Morcadès, toujours soeur d'Arthur. Sur Bellurepar, voir J.L.A. HUILLARD-BREHOLles, Historia diplomatica Frederici secundi, V, 1, Paris 1857, p. 411; 5 octobre 1239.

${ }^{26}$ Archivio Segreto Vaticano, Instrumenta Miscellanea, 7205; pouillé des redevances dues à Patti, vers 1370 , le plus ancien document sur Gioiosa.

${ }^{27}$ Antoine de la SAlle, La Salade, éd. Fernand Desonay, Liège-Paris 1935, pp. 63-130.

${ }^{28}$ Gaston PARIS, «La légende du Tannhaüser», Légendes du Moyen-Age, Paris 1903, pp. 113-145. 
du sabbat, les belles dames s'y transforment en serpents ${ }^{29}$. Cette légende, les chevaliers allemands la recherchent partout, Dietrich de Niem, près de Puzzoles, dans le milieu infernal d'un mont volcanique, qu'il nomme "Der Gral»; Frère Félix Faber, plus érudit, à Chypre, en 1485, dans les environs de Paphos, et c'est maintenant le Venusberg. Depuis 1453, en effet, le poète Hermann de Sachsenheim a cristallisé la légende de la montagne enchantée et maudite autour de Vénus et de son époux, le Tanhäuser, ce Minnesinger contemporain de Frédéric II et de ses luttes, tandis que le pape borné et damné devient Urbain IV, l'ennemi des Hohenstaufen.

La figure ambiguë de la fée domine, sans conteste, un monde sensuel et infernal qui fascine la chevalerie européenne, et surtout allemande; ell s'épanouit dans la chanson de geste tardive et le roman courtois, sans que sa luxure et le péril qu'ils courent effraient trop les chevaliers; fascinée aussi, la culture cléricale s'en sert comme d'une épouvantail. Dans la formation de sa légende, le royaume normand de Sicile, de culture française, a sans doute joué un rôle essentiel, avant que l'invasion allemande et l'offensive des cleres ne contribuent au transfert de ses localisations et à la dissociation des deux aspects que la légende d'Arthur unissait d'abord: le paradis insulaire verdoyant et la malédiction infernale. La littérature courtoise n'a gardé que le premier, innocent et sensuel, dominé par Morgane; la littérature religieuse n'a conservé que le second, infernalisant Arthur. L'hypothèse que, dès le xnle siècle, l'Etna était ce paradis maudit, est vraisemblable: on sait que la légende arthurienne a été portée en Italie, avant même la pénétration des oeuvres de Geoffroy de Monmouth et de Chrétien de Troyes, par les chanteurs. En Sicile, une part notable de l'aristocratie française et quelques évêques étaient bretons et les relations avec l'Angleterre très étroites. L'Etna a pu cumuler ses prestiges de montagne magique, ceux

29 Le serpent, la «biscia», est aussi la forme que prend la fille de Morgane, la Ponzela Gaia, dans un conte du XIve siècle italien; R.M. RUGGIERI, "Avventure di caccia nel regno di Artù», Mélanges Rita Lejeune II, Gembloux 1969, pp. II03-II20. 
de l'Antiquité des mythographes, ceux des légendes populaires qui s'attachent aux êtres maudits enchaînés sous les volcans, géants ou damnés, et ceux enfin qu'il a pu ravir au Mont Eryx, que le Moyen-Age, même érudit, ne reconnaît guère dans le Djabal Hamid et le Monte San Guiliano.

La présence d'Arthur, qui appelait celle de sa soeur Morgane, ancrait la Sicile reconquise dans la géographie européenne imaginaire. Excalibur consacrait un embryon de mythe de fondation, rattachant la monarchie normande non tant au passé glorieux des Bretons qu'à l'avenir immortel de leur prince. Etrange patronage que celui de l'hôtesse qui est reine dans l'île où Arthur est accueilli, et qui va se fixer à la fois dans la littérature et dans le folklore: de Reggio, dans le mirage de la Fatamorgana ${ }^{30}$, la Sicile apparaît comme son royaume enchanté. Fée et "déesse»" , savante et magicienne, Morgane est venue dans l'île portée par la seule tradition orale, qui cumule sur elle les aspects positifs et négatifs: contaminée par le thème du serpent ${ }^{32}$, elle est aussi la dame des "Vaus-sanz-retour», «aspirée et de luxure et de dyable». Le mystère est dans le choix de cette maîtresse ambivalente; il est au coeur de la légende arthurienne.

\section{Fascination et ambiguité: un paradis incestueux?}

Si le mythe d'un Arthur fondateur ou protecteur de la monarchie sicilienne ne s'est pas enraciné, bousculé par le tourbillon politique des Teutoniques, la présence prolongée de Mor-

${ }^{30}$ Le phénomène de mirage, signalé sous le nom de Morgane, «sirène», dès 1658 par PLACIDO REYNA, Delle Notizie istoriche della città di Messina, trad. latine in GRAEvrus, Thesaurus Antiquitatum et historiarum Siciliae, IX, Leyde 1723, p. 36, affecte le détroit de Messine.

${ }^{31}$ "Dea phantastica» pour Giraud le cambrien, et dans l'oeuvre anglaise, Sir Gawain and the Green Knight; cf. P. Merrens-Fonck, «Morgan, fée et déesse», Mélanges rita Lejeune II, Gembloux 1969, pp. 1067-1076.

${ }^{32}$ Peut-être y a-t-il un rapport entre sa seigneurie sur la montagne et les serpents ailés du Mont Etna qui se nourrissent d'enfants, du Livre des Merveilles (ms. fr. 2810, BN), édité par HeNRI OMONT. Paris 1907, pl. 128. 
gane pose sur l'île comme un mirage, charmant, mais trouble. Car le lien qui l'unit, ou plutôt l'a unie, à Arthur, c'est aussi l'inceste. Cette soeur est d'abord une marraine qui aide Arthur à naître, à la vie terrestre, dans une naissance enchantée et féerique, et à l'immortalité, terrestre encore, dans un véritable enlèvement ${ }^{33}$. Mais il est de sa nature de fée de ne pas respecter les lois humaines et divines. Cette soeur (qui n'est pas toujours appelée Morgane, tandis que Morgane n'a pas toujours été la soeur d'Arthur) a donc été l'amante d'Arthur, par le choix volontaire du public, suivi par les littérateurs, que la tradition ne contraignait pas; et la tradition se crée et ce consolide qui fera d'eux les parents de Mordred ${ }^{34}$. Le silence qui pèse sur Morgane et l'insistance de Gervais sur Mordred sont, je le crois, un indice du malaise sous-jacent; le non-dit, ici, pèse lourd, quand tous les publics, le sicilien comme celui de Gervais, étaient au courant du redoutable mystère.

La malédiction de l'inceste est peut-être un simple retournement d'une antique hiérogamie celtique sous l'influence chrétienne. C'est peut-être, plus modestement, la recherche d'une légende explicative, par le châtiment divin, à la chute du royaume breton et de son hégémonie universelle. Quelle que soit son origine, c'est sa présence secrète dans le filon des contes siciliens qui me semble justifier cette étonnante similitude entre Arthur, «méhaigné», blessé aux jambes ou aux hanches, roi pécheur, et pour cela blessé et vaincu, et un autre roi pêcheur, lui aussi étendu sur un lit, en punition d'un autre péchés ${ }^{35}$. Paradis païen, l'Etna offre à la verte vallée des plaisirs un cadre infernal, brûlé et brûlant, qui augure mal de son choix. La littérature courtoise s'en accomodera, peut-être aidée en cela par l'éloignement; la

${ }^{33}$ Cf. Marie Claude Blanchet, «L'Argante de Layamon", Melánges J. Frappier I, Genève 1970, pp. 133-144.

${ }^{34}$ Charles Mel.a, dans son compte-rendu du film Excalibur de John Boorman, "Un cinéaste médiéval», l'Ane 2, 1981, p. 49, insiste à bon droit sur la lucidité du cinéaste, qui concrétise un courant de fantasmes qui serpente dans toute la littérature arthurienne.

${ }^{35}$ Là encore, John Boorman fait éclater l'évidence d'une identité cachée, souterraine: Arthur est le roi pêcheur. 
littérature des exempla saisira l'occasion d'une infernalisation moralisatrice.

Il reste à poser la question la plus angoissante: pourquoi, dans la légende arthurienne, la Sicile normande a-t-elle choisi la part la plus difficile, la plus noire, la plus ambiguë surtout? Peut-être était-ce la seule localisation capable d'intégrer l'île à l'épopée chevaleresque par excellence. La sensualité de Morgane, si sensible au XII siècle, est-elle un rapport avec les fantasmes des conquérants francs mis en relation avec la société musulmane dont la morale, différente, pouvait paraître plus accueillante aux désirs des puissants? Ou bien, en accord plus profond avec la vision de Pierre de Blois, révèle-t-elle défiance et pessimisme des conquérants à l'égard du monde dangereux et inquiétant de leur conquête? «Mère des géants et nourrice des tyrans", l'île n'avait pas bonne presse et ce monde exotique et féroce s'accordait déjà par anticipation avec l'image enchantée et violente de la chevalerie arthurienne.

Excalibur allait finir quelque part dans les salles de la Hofburg, à Vienne, parmi les trophées d'Henri VI; toute une tradition sombrait avec l'invasion teutonique et toute une culture, qui ne sera pas aisément remplacée. Cette arme magique nous restitue la profondeur mythique encore mal explorée de la puisance française dans l'île, une sensibilité trouble et inquiétante qui débouche sur des tentatives de constructions complexes, milittéraires, mi-folkloriques, aptes à donner un sens, une origine, un passé présent, à la domination féodale. 